\title{
Nature of X-ray Sources From the Field of Nearby, Poor Cluster of Galaxies
}

\author{
M. Hudaverdi $\mathbf{i}^{1,2} \dagger$, H. Kunieda ${ }^{1}$, and E.N. Ercan ${ }^{2}$ \\ ${ }^{1}$ Department of Physics, Nagoya University, Furocho, Chikusa, 4648602, Japan \\ email: hudaverd@u.phys.nagoya-u.ac.jp, kunieda@u.phys.nagoya-u.ac.jp \\ ${ }^{2}$ Department of Physics, Boğaziçi University, Bebek, 34342, Istanbul, Turkey \\ email: ercan@boun.edu.tr
}

\begin{abstract}
A194 and A1060 are studied for the nature of point source properties. X-ray to optical luminosity ratios $(\mathrm{Lx} / \mathrm{Lb})$ are evaluated for selected galaxies. Cumulative $\log (\mathrm{N})-\log (\mathrm{S})$ and intrinsic luminosity functions are applied for a comparative evaluation with Lockman Hole. 46 and 32 X-ray point sources are detected by EPIC-PN, for A194 and A1060 respectively. The XLF space density shows significant excess compared to field and local group space density. The result is interpreted as the fueling of AGNs as they enter the ICM from outskirts of the clusters and dimming of the very bright AGNs as they further proceed into cluster core.
\end{abstract}

Keywords. Clusters of galaxies, individual; A194, A1060

\section{Analysis and Results}

We studied the X-ray source properties of two nearby $(\mathrm{z}<0.02)$ clusters of galaxies A194 and A1060 and compare our findings to the non-cluster fields in order to understand the nature of point sources in the cluster environment. A multi-band source detection technique is applied. X-ray to optical luminosity ratios $(\mathrm{Lx} / \mathrm{Lb})$ are evaluated for selected galaxies. We detect $78 \mathrm{X}$-ray point sources by EPIC-PN in total. The integrated $\log (\mathrm{N})$ $\log (\mathrm{S})$ distributions estimate a significantly higher number of sources in cluster field at the flux limit of $F_{X}=10 \mathrm{E}-14 \mathrm{ergs} \mathrm{cm}^{-2}$. Based on the estimated X-ray luminosity function (XLF) space density of the sources, we found a significant excess compared to field and local group space density. The luminosity range is really faint $(39.6<\log (\mathrm{Lx})$ $<40.8 \mathrm{ergs} / \mathrm{s}$ ), which is possibly contaminated by LXMBs, hot halo, star forming and low luminosity active galactic nuclei (LLAGN). Based on our results, we say that the $\mathrm{x}$-ray emission is induced by infall as also reported by Chandra observations (Molnar et al. 2002 and Martini et al. 2002) which may also explain that the brighter members locates at the outskirt of cluster. When we look at the XLF from the brighter end we see that our sample is lack of very luminous AGNs. Since our clusters are nearby, only the central region is covered. The absence of bright AGNs could be explained as the galaxies further fall into central regions they quench and get fainter (Martini et al. 2002).

\section{References}

Martini P., Kelson D. D., Mulchaey J., \& Trager S. C., 2002, ApJ, 576, L109.

Molnar, S. M., Hughes, J. P., \& Donahue, M., et al. 2002, ApJ, 573, 91.

$\dagger$ The author acknowledges the funding from Boğaziçi University Research Foundation coded 06HB301 and COE of Nagoya University. 